\section{Tim-3 promotes maternal tolerance}

Natural killer (NK) cells have a protective role in establishing and maintaining maternal tolerance to a developing fetus. In Science Signaling, Li et al. show that peripheral NK cells upregulate their expression of the immunoinhibitory molecule Tim-3 during the first trimester of pregnancy and thereby promote maternal-fetal tolerance via interaction with the Tim-3 ligand galectin-9. Tim$3^{+}$NK cells express anti-inflammatory cytokines and are less cytotoxic than Tim-3- NK cells. NK cells from women who have experienced recurrent miscarriages have lower expression of Tim-3 protein and are more cytotoxic than NK cells obtained during a normal pregnancy. Corresponding differences at the chromatin level and in gene expression are also seen in Tim $-3^{+}$and Tim$3^{-}$NK cells. Tim- $3^{+}$NK cells also contribute to an increased frequency of induced regulatory $T$ cells dependent on the cytokine TGF- $\beta 1$, which suggests another mode by which NK cells contribute to maternal-fetal tolerance.

Sci. Signal. 10, eaa4323 (26 September 2017)

\section{CD28 enhances mitochondrial function}

Costimulation via the co-receptor $\mathrm{CD} 28$ is required for the generation of effective memory T cells. A lack of CD28 signaling during the priming of naive $\mathrm{T}$ cells results in anergic $\mathrm{T}$ cells that fail to provide protection against subsequent challenge. In Cell, Pearce and colleagues reveal that CD28 signals at initial priming lead to metabolic changes that result in the increased mitochondrial morphology, spare respiratory capacity and fattyacid metabolism necessary for effector memory responses. Mechanistically, CD28 transiently suppresses the metabolic regulator TXNIP, which regulates expression of the microRNA miR-33. In turn, CD28 enhances expression of the miR-33 target Cpt1a, which encodes the rate-limiting mitochondrial enzyme carnitine palmitoyltransferase that is necessary for fatty-acid oxidation. Modulation of miR-33 expression during $\mathrm{T}$ cell priming is inversely correlated with memory-cell function after recall challenge. The Cptla inhibitor etomoxir likewise impairs the CD28-dependent function of memory T cells. Thus, early CD28 signals prepare T cells for subsequent memory responses by enhancing mitochondrial capacity and fatty-acid utilization.

$L A D$ Cell (14 September 2017) doi:10.1016/j.cell.2017.08.018

\section{What is the point of the gallbladder?}

The lectin and surfactant SP-D is a secreted component of the innate immune system known mainly for its presence and function in the lungs. In the Proceedings of the National Academy of Sciences USA, Taniguchi and colleagues find that SP-D is also generated by epithelial cells in the gallbladder, but nowhere else within the digestive system or in the liver. SP-D is secreted into the intestine as a component of bile; once there, it binds particular commensals such as Lactobacillus species. Deficiency in SP-D results in dysbiosis, including a reduction in the abundance of Clostridia species, which have been linked to the homeostasis of regulatory T cells. SP-D-deficient mice also exhibit worse experimentally induced colitis. The apparently unique production of SP-D within the digestive system has potentially important implications for otherwise routine gallbladder removal (cholecystectomy).

Proc. Natl. Acad. Sci. USA (19 Sep 2017) doi:10.1073/

pnas. 1712837114

\section{Old fat macrophages}

Catecholamine signaling is normal in the elderly; however, the catecholamine-induced generation of free fatty acids diminishes with age. In Nature, Camell et al. show that adipose tissue macrophages regulate the age-dependent decrease in adipocyte lipolysis in mice by lowering the availability of noradrenaline. Aged adipose tissue macrophages inhibit the release of free fatty acids from noradrenaline-stimulated young adipocytes and have high expression of enzymes that regulate catecholamine catabolism, such as monoamine oxidase A, as well as of genes encoding molecules linked to inflammasome activation. Aged $\mathrm{Nlrp}^{-3^{--}}$mice show restoration of expression of factors involved in catecholamine catabolism and in the release of free fatty acids after fasting, which are lower in old wild-type mice than in young wild-type mice. Inhibition of monoamine oxidase A in inflammasome-activated macrophages restores lipolysis in noradrenalineinduced adipocytes in vitro and fasting-induced lipolysis in old mice in vivo. Macrophages are present in association with sympathetic nerve fibers in the adipose tissue, which suggests that they might regulate the access of adipocytes to noradrenaline.

Nature (27 September 2017) doi:10.1038/nature23912

\section{Breathe easy with neutrophils}

The lungs are continually exposed to harmful pathogens such as Aspergillus fumigatus, and prompt clearance of such pathogens with limited inflammation is needed to avoid dangerous aspergillosis or collateral damage from the immune response. In Science, Hohl and colleagues demonstrate that myeloid cells in the lungs, such as neutrophils, induce an apoptosis-like process of programmed cell death (PCD) in A. fumigatus conidia. This process is dependent on the generation of reactive oxygen species in neutrophils via the NADPH complex. Accordingly, fungi that overexpress the anti-apoptotic protein AfBIR1 are relatively resistant to neutrophil-induced $P C D$ and demonstrate enhanced virulence. This previously unknown PCD-dependent mechanism for the clearance of conidia represents an effective form of lung immunosurveillance that prevents transition of the fungus to its invasive hyphal form; additionally, it suggests a potential target for the pharmacological treatment of fungal infection.

Science 357, 1037-1041 (2017)

\section{Neurodegenerative signature}

Microglia lose their homeostatic function during neurodegenerative disorders. In Immunity, Butovsky and colleagues show that in mouse models of amyotrophic lateral sclerosis, Alzheimer's disease and multiple sclerosis, the neurodegenerative phenotype of microglia is triggered by activation of the receptor TREM2-apolipoprotein E (APOE) pathway. This phenotype is characterized by the suppression of TGF- $\beta$-dependent genes encoding homeostatic molecules, such as Sall1 and Tgfbr1, and the upregulation of genes encoding inflammatory molecules, including Apoe and Clec7a, and is distinct from microglial activation induced by lipopolysaccharide or the cytokine IFN- $\gamma$. The expression of APOE and Clec7a increases in microglia situated in close proximity to amyloid-b plaques in humans and mice and is induced by the injection of apoptotic neurons but not by Escherichia coli or zymosan. The neurodegenerative phenotype is not induced in Apoe $\mathrm{e}^{-/-}$or Trem $2^{-/-}$microglia after the phagocytosis of apoptotic neurons, and in contrast to wild-type microglia, Apoe ${ }^{-/-}$microglia from the spinal cord of mice at the peak of experimental autoimmune encephalomyelitis suppress T cell proliferation. As such, APOE might inhibit a tolerogenic phenotype in microglia.

Immunity 47, 566-581 (2017) 